\title{
TOXOPLASMA GONDII: ÉTUDE ULTRASTRUCTURALE DES FORMATIONS KYSTIQUES OBSERVÉES EN CULTURE DE FIBROBLASTES HUMAINS
}

\author{
M. L. DARDÉ*, B. BOUTEILLE*, M. J. LEBOUTET**, \\ A. LOUBET**, M. PESTRE-ALEXANDRE*
}

\begin{abstract}
RÉSUMÉ. L'inoculation de cultures de fibroblastes humains par une souche de Toxoplasma gondii non pathogène pour la souris (souche Prugniaud, Limoges, 1964) donne naissance à des formations d'aspect kystique. Leur ultrastructure est comparée d'une part, à celle des kystes cérébraux chez la souris, d'autre part, pour la même souche de $T$. gondii, à celle des colonies intracellulaires de tachyzoïtes obtenues dans les cellules sarcomateuses TG 180 de souris.

La microscopie électronique révèle les similitudes entre les formations kystiques observées in vitro et les kystes cérébraux observés chez la souris.
\end{abstract}

Mots-clés : Toxoplasma gondii. Ultrastructure. Culture cellulaire. Fibroblastes. Kystes. Cellules TG 180.

\section{Toxoplasma gondii: Ultrastructure of cyst-like forms observed in human fibroblasts cell culture.}

SUMMARY. A strain of Toxoplasma gondii, non pathogenic to mice (Prugniaud strain) produced cyst-like stages when inoculated in human fibroblast culture.

An ultrastructural study was performed in order to compare these cyst-like forms to brain cysts of the same strain and to intracellular clusters of tachyzoites observed in mice TG 180 sarcoma cells.

Electron microscopy confirmed the similarities between brain cysts and cysts obtained in vitro.

Key-woras: Toxoplasma gondii. Ultrastructure. Cell culture. Fibroblasts. Cysts. TG 180 sarcoma cells.

L'infestation d'une culture cellulaire de fibroblastes humains par des toxoplasmes d'une souche chronique chez la souris fait apparaître différents aspects évolutifs : des colonies intracellulaires de tachyzoïtes détruisant rapidement la cellule et des formations évoquant des kystes tissulaires. L'ultrastructure

* Service de Parasitologie.

** Service d'Anatomie pathologique I.

CHU Dupuytren, F 87042 Limoges Cedex.

Accepté le 15 septembre 1989. 
de ces différentes formes, observée en microscopie électronique, est comparée à celle des kystes cérébraux et des colonies de tachyzoïtes obtenues dans les cellules sarcomateuses TG 180 d'ascite de souris. Cette étude est en faveur de la présence de véritables kystes de toxoplasmes en culture cellulaire de fibroblastes humains.

\section{Matériel et méthodes}

Souche de TOXoplasme utilisée

La souche de T. gondii utilisée est un clone dérivé de la souche Prugniaud, isolée à Limoges en 1964 d'un enfant mort de toxoplasmose congénitale (Martrou et al., 1965). Depuis l'isolement, elle est maintenue sur souris Swiss par inoculation intrapéritonéale, tous les 12 mois, de suspensions de cerveaux de souris infectées. Le clonage a été réalisé par isolement d'un kyste par micromanipulation selon une méthode antérieurement décrite (Dardé et al., 1988a).

\section{DifFÉRENTES FORMES ÉTUdiÉES EN MICROSCOPIE ÉLECTRONIQUE}

Kystes intracérébraux.

Les kystes intracérébraux proviennent de cerveaux de souris inoculées trois mois auparavant par des kystes de T. gondii.

Tachyzoütes.

Les tachyzoïtes sont obtenus par multiplication in vivo dans les cellules sarcomateuses TG 180 de souris, selon une méthode préalablement décrite (Desmonts et Remington, 1980 ; Dardé et al., 1988b). Pour l'étude ultrastructurale, l'ascite de souris est récupérée 72 heures après l'inoculation intrapéritonéale conjointe de cellules TG 180 et de tachyzoïtes. Elle est constituée de cellules sarcomateuses contenant des amas de toxoplasmes, visibles au microscope à contraste de phase $(\times 400)$.

Toxoplasmes en culture de fibroblastes humains.

Des cultures de fibroblastes humains (Flow 2002, Flow Laboratories) sont effectuées en flacon de $75 \mathrm{~cm}^{2}$ (Falcon ${ }^{\circledR}$, Beckton-Dickinson) dans du milieu MEM additionné de $10 \%$ de sérum de veau fœtal (Gibco) et placées à $37^{\circ} \mathrm{C}$ sous $5 \%$ $\mathrm{CO}_{2}$. Après inoculation des toxoplasmes, la proportion de sérum de veau fœetal est ramenée à $1 \%$ afin de limiter la croissance cellulaire. Ce milieu est renouvelé tous les 2 jours.

Les cultures sont infestées soit avec des tachyzoïtes $\left(10^{5}\right.$ par flacon) soit avec des kystes cérébraux (environ 30 kystes par flacon) isolés en gradient de Percoll selon la méthode de Blewett (1983). La fixation pour étude ultrastructurale est pratiquée 15 jours après l'infestation. 


\section{Microscopie ÉLECTRONIQUE}

Pour la microscopie électronique, les différentes formes étudiées, après fixation par la glutaraldéhyde à 2,5\% subissent une post-fixation par le tétra-oxyde d'Osmium à $2 \%$, et sont incluses en Épon. Le contraste est assuré par une coloration à l'acétate d'uranyl-citrate de plomb. Les coupes ultrafines sont examinées au microscope électronique Zeiss EM 9.

\section{Résultats}

\section{KyStes INTRACÉRÉBRAUX}

Les résultats de notre étude ultrastructurale des kystes cérébraux observés chez la souris sont en accord avec ceux déjà décrits par d'autres auteurs pour ces formations (Ferguson et Hutchison, 1987). Le kyste cérébral est intracellulaire (fig. 1 et 2). La cellule hôte est délimitée par une membrane plasmique et son cytoplasme contient des organites identifiables (mitochondries, reticulum endoplasmique) qui attestent de sa viabilité. La paroi du kyste est limitée par une membrane festonnée sur la face interne de laquelle s'accumule, de façon irrégulière, un précipité granulaire dense aux électrons. Un précipité granulaire plus dispersé remplit l'espace entre les toxoplasmes (fig. 2). Les toxoplasmes ont l'aspect de bradyzoïtes (fig. 2 et 3) caractérisé par une grande richesse en vacuoles polysaccharidiques et en micronèmes, des rhoptries à structure dense, un noyau postérieur.

\section{Colonies de tachyzoites dans les Gellules TG 180}

Les parasites sont contenus dans une vacuole parasitophore limitée par une fine membrane (fig. 4 et 5). Dans le cytoplasme de la cellule hôte (fig. 5), du reticulum endoplasmique bordé de ribosomes s'accumule souvent contre la membrane vacuolaire. Les ribosomes sont absents du côté du reticulum endoplasmique adjacent à la vacuole parasitophore. Le contenu de la vacuole parasitophore entre les toxoplasmes est occupé par quelques formations membranaires et tubulaires. Il n’y a pas de précipité granulaire. Les parasites ont les caractéristiques des tachyzoïtes : peu de granules polysaccharidiques, peu de micronèmes, des rhoptries dont les extrémités postérieures ont une structure en nid d'abeille, un noyau en position médiane (fig. 5).

\section{Toxoplasmes EN GUlture DE fibroblastes}

\section{Évolution de l'infection.}

Quel que soit le matériel inoculé (tachyzoïtes ou bradyzoïtes), l'évolution de l'infection observée au microscope inversé $(\times 100)$ est la même : des amas 

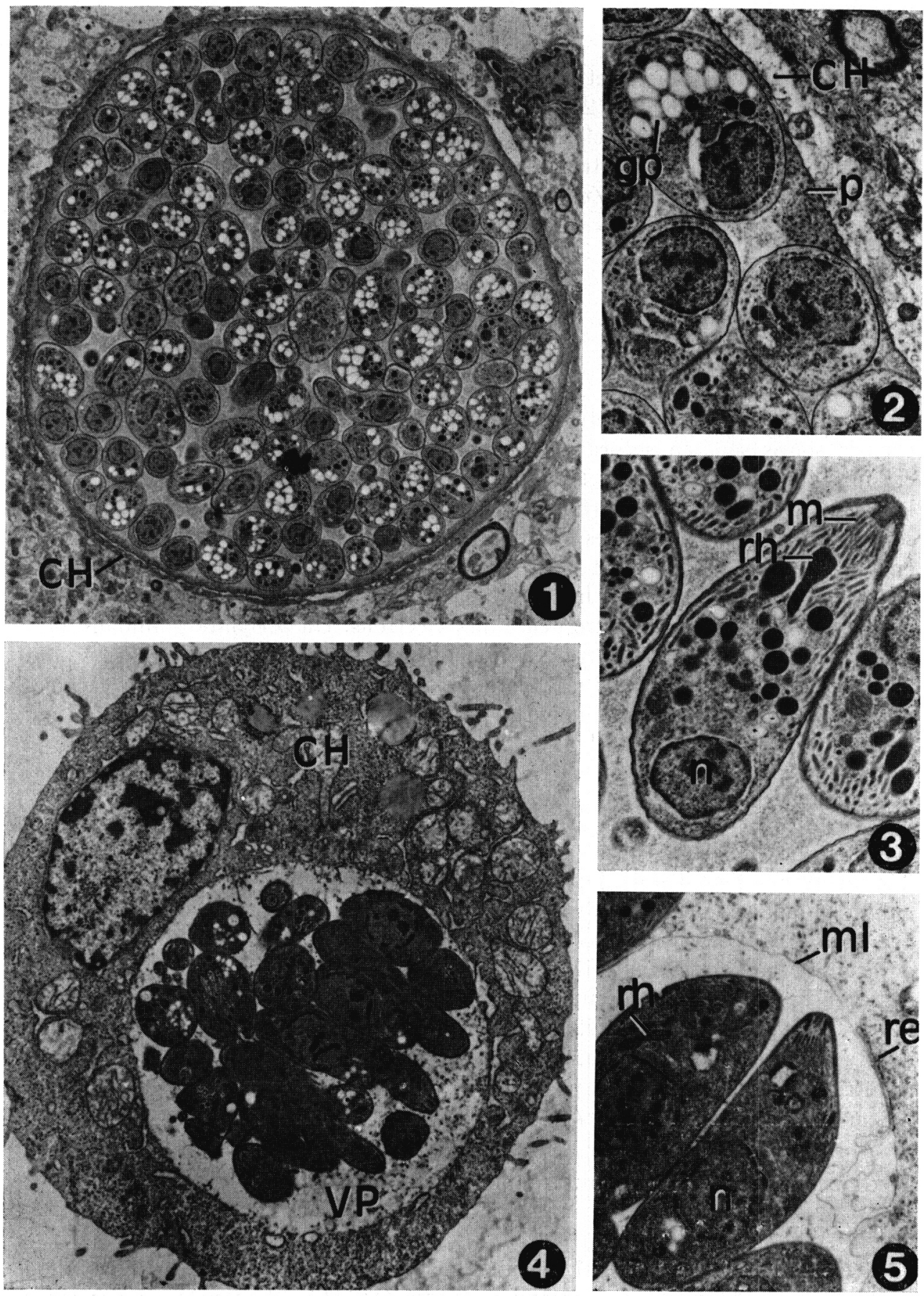

FIg. 1 à 5 . 
arrondis de toxoplasmes apparaissent dans les fibroblastes dès le $6 \mathbf{e}$ jour après inoculation de tachyzoïtes, dès le $10^{\mathrm{e}}$ jour après inoculation de bradyzoïtes. Certaines cellules hôtes sont alors détruites et des parasites libres sont observés au sein de petites plages de lyse cellulaire, traduisant une multiplication rapide de certains éléments. Des amas de toxoplasmes intracellulaires d'aspect différent sont aussi observés. Ils sont entourés d'une paroi réfringente épaisse et peuvent atteindre 100 à $200 \mu \mathrm{m}$ de diamètre. Ils persistent à l'intérieur de la cellule tant que celle-ci reste adhérente au support. Ces formations d'aspect kystique ont pu être observées dans les fibroblastes jusqu'à 2 mois après l'inoculation.

\section{Aspects ultrastructuraux.}

Les deux aspects coexistant au sein de la culture de fibroblastes humains sont décrits en ultrastructure.

Le premier aspect est celui des colonies intracellulaires de tachyzoïtes, parfois volumineuses, présentant les mêmes caractéristiques que celles observées pour les colonies de tachyzoïtes dans les cellules TG 180 (fig. 6). La vacuole parasitophore est limitée par une membrane mince, marquée, par endroits, par de longues invaginations tubulaires (fig. \%). L'espace entre les toxoplasmes, dépourvu de substance granulaire dense aux électrons, ne comporte que quelques formations membranaires. Les toxoplasmes ont toutes les caractéristiques déjà citées des tachyzoïtes. Plusieurs présentent des aspects d'endodyogénie.

Le second aspect ( $f$ ig. 8 et 9) est celui de colonie intrafibroblastique occupant presque la totalité du cytoplasme de la cellule et présentant toutes les caractéristiques d'un vrai kyste toxoplasmique. La cellule hôte reste viable comme l'atteste la présence d'organites cytoplasmiques identifiables (reticulum endoplasmique, ribosomes). La paroi du kyste est identique à celle des kystes cérébraux. Elle est constituée d'une membrane irrégulière épaissie par le dépôt sur sa face interne d'un précipité granulaire condensé en une couche homogène. Une substance granulaire dense aux électrons remplit l'espace entre les toxoplasmes. Les parasites, à ce stade, ont encore quelques éléments évocateurs de tachyzoïtes (rhoptries

FIG. 1. - Kyste intracérébral de $T$. gondii contenant des bradyzoïtes riches en granules polysaccharidiques ( $\times 2$ 925).

FIG. 2. - Kyste intracérébral : détail de la paroi kystique et du cytoplasme de la cellule hôte ( $\times 9500)$.

FIG. 3. - Kyste intracérébral : détail d'un bradyzoïte avec un noyau postérieur, des rhoptries denses et de nombreux micronèmes ( $x$ 11 500).

FIG. 4. - Colonie de tachyzoïtes dans une vacuole parasitophore de cellule sarcomateuse TG $180(\times 7300)$.

FIg. 5. - Détail d'une vacuole parasitophore dans une cellule TG 180. A noter la finesse de le membrane limitante, bordée par le reticulum endoplasmique de la cellule hôte, et les tachyzoïtes (rhoptries en nid d'abeille, peu de micronèmes et de granules polysaccharidiques) ( $\times 9500)$.

CH : cellule hôte ; gp : granules polysaccharidiques ; $\mathrm{m}$ : micronèmes ; $\mathrm{ml}:$ membrane limitante ; n : noyau; p : paroi kystique ; re : reticulum endoplasmique ; rh : rhoptries ; VP : vacuole parasitophore. 

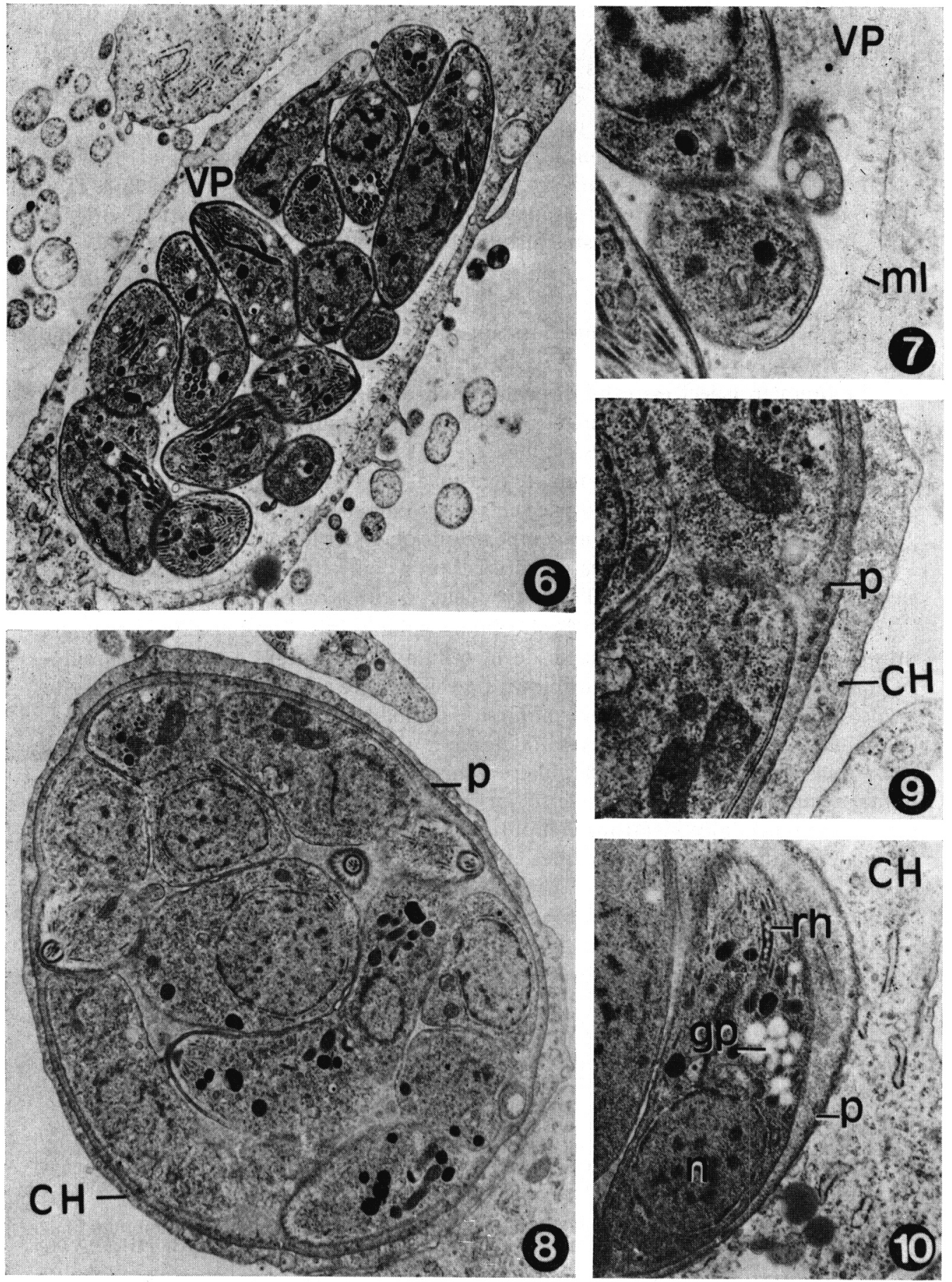

FIG. 6 à 10. 
avec une structure en nid d'abeille) mais le nombre de granules polysaccharidiques. et de micronèmes est plus élevé, le noyau est plus postérieur (fig. 10).

\section{Discussion}

L'étude ultrastructurale des cultures de fibroblastes humains infectés par une souche de toxoplasmes non pathogène pour la souris révèle la présence à l'intérieur de certains fibroblastes de véritables kystes de $T$. gondii. Ces formations kystiques sont similaires aux kystes cérébraux de souris. Leur description rejoint celle des études ultrastructurales portant sur les kystes tissulaires (Mehlborn et Frenkel, 1980 ; Ferguson et Hutchison, 1987) : formation intracellulaire limitée par une membrane aux invaginations plus ou moins nombreuses et profondes selon l'ancienneté du kyste, soulignée sur sa face interne par une accumulation de substance granuleuse. Un matériel granulaire plus dispersé occupe l'espace entre les toxoplasmes. La richesse en granules polysaccharidiques et en micronèmes est en faveur d'une évolution vers un stade bradyzoïte, mais la présence de rhoptries à structure lâche, telles qu'elles sont décrites chez les tachyzoïtes, témoigne d'une activité sécrétrice persistante au sein de ces jeunes kystes.

Pour désigner ces formations, le terme de "kyste " semble préférable à celui de "pseudokyste ". Le terme de "pseudokyste " peut être à l'origine de confusion car il est utilisé pour désigner, selon les auteurs, tantôt les formations observées dans les infections chroniques, tantôt les amas de tachyzoïtes intracellulaires (Frenkel, 1973). Or, l'aspect ultrastructural de ces kystes observés en culture cellulaire s'oppose sur de nombreux points aux vacuoles parasitophores contenant des amas de tachyzoïtes observés dans les cellules sarcomateuses TG d'ascite de souris infectée et, en culture cellulaire, dans une forte proportion de fibroblastes infectés. Les caractères ultrastructuraux de ces vacuoles contenant des colonies de tachyzoïtes sont en accord avec ceux décrits par d'autres auteurs (Nichols et O’Connor, 1980; Ferguson et Hutchison, 1987) : vacuole parasitophore limitée par une membrane mince, marquée par de longues invaginations tubulaires, contenant des formations membranaires dispersées entre les tachyzoïtes.

La possibilité de formation de kystes de toxoplasmes in vitro, a déjà été

FIG. 6. - Colonie de tachyzoïtes dans une vacuole parasitophore de fibroblaste en culture cellulaire $(\times 7600)$.

FIG. 7. - Vacuole parasitophore dans un fibroblaste en culture cellulaire : détail de la membrane limitante ( $\times 21500)$.

Fig. 8. - Kyste intrafibroblastique en culture cellulaire ( $\times 9000)$.

Fig. 9. - Détail de la paroi du kyste intrafibroblastique de la figure 8. A noter l'épaisseur de la paroi, la substance granulaire intrakystique, le cytoplasme de la cellule hôte $(\times 20000)$.

Fig. 10. - Toxoplasme observé dans un kyste intrafibroblastique

et présentant plusieurs caractéristiques de bradyzoïtes ( $\times 11500)$.

$\mathrm{CH}$ : cellule hôte ; gp : granules polysaccharidiques ; $\mathrm{m}:$ micronèmes ; $\mathrm{ml}:$ membrane limitante ; $\mathrm{n}$ : noyau ; p : paroi kystique ; re : reticulum endoplasmique ; rh : rhoptries ; VP : vacuole parasitophore. 
rapportée par plusieurs auteurs (Matsubayashi et Akao, 1963 ; Hoff et al., 1977 ; Rondanelli et al., 1981 ; Jones et al., 1986).

Outre les études ultrastructurales, des méthodes biologiques ont permis de prouver la réalité de la présence de bradyzoïtes. Hoff et al. (1977) démontrent l'existence de bradyzoïtes dès le $6^{\mathrm{e}}$ jour, après inoculation de culture de fibroblastes de singe, en observant la durée de la période pré-patente d'excrétion des oocystes après ingestion par le chat des cultures infectées. Cette période pré-patente n'est en effet que de 3 à 10 jours pour les bradyzoïtes alors qu'elle est de 20 à 48 jours pour les tachyzoïtes.

Les facteurs favorisant la formation des kystes in vitro ne sont pas connus.

- Le rôle de la souche de T. gondii semble important : les kystes n'apparaissent in vitro qu'avec des souches donnant des kystes tissulaires chez la souris dans les conditions habituelles d'inoculation, de telle sorte que certains auteurs (Rondanelli et al., 1977) ont proposé d'en faire un marqueur de la virulence des souches.

- La nature de la cellule hôte n'apparaît pas fondamentale : les kystes ont été décrits in vitro dans des cellules Hela (Matsubayashi et Akao, 1963), des cellules VERO de rein de singe (Rondanelli et al., 1977), des fibroblastes de singes, de souris, des fibroblastes humains (Schwartzman, 1987), des astrocytes (Jones et al., 1986). In vivo, la localisation préférentielle des kystes dans les neurones et les cellules musculaires pourrait être due au fait qu'il s'agit de cellules à longue durée de vie dans lesquelles le kyste peut rester longtemps à l'abri des phénomènes immunitaires (Ferguson et Hutchinson, 1987).

- Les facteurs immunitaires ne sont pas indispensables à la formation des kystes in vitro (Hoff et al., 1987). Cependant, Jones et al. (1986) ont montré que le traitement par l'interféron $\gamma$ des cultures d'astrocytes infectées permet la persistance des kystes pendant des périodes prolongées, en évitant, par ailleurs, la destruction des cellules par les tachyzoïtes.

En conclusion, il faut souligner la coexistence au sein d'une même culture, et donc dans les mêmes conditions d'environnement, de deux types de formations toxoplasmiques aisément différenciables : d'une part, des colonies de tachyzoïtes contenues dans une vacuole parasitophore à membrane mince, se multipliant rapidement et détruisant la cellule hôte, d'autre part, des kystes persistant plusieurs semaines dans la cellule hôte, sans la détruire. La coexistence de ces deux comportements pose le problème d'une relation hôte-parasite particulière ou de déterminismes génétiques différents au sein de la population de toxoplasmes.

\section{RÉFÉRENCES}

Blewett D. A., Miller J. K., Harding J. : Simple technique for the direct isolation of toxo plasma tissue cysts from foetal ovine brain. Vet. Record., 1983, 112, 98-100.

Dardé M. L., Bouteille B., Pestre-Alexandre M. : Analyse iso-enzymatique de souches clonées de Toxoplasma gondii. Bull. Soc. Fr. Parasitol., 1988a, 6, 41-46.

dardé M. L., Bouteille B., Pestre-Alexandre M. : Isoenzymic characterization of seven strains of Toxoplasma gondii by isoelectrofocusing in polyacrylamide gels. Am. J. Trop. Med. Hyg., 1988b, 39, 551-558. 
Desmonts G., Remington J. S. : Direct agglutination test for diagnosis of Toxoplasma infection: method for increasing sensitivity and specificity. J. Clin. Microb., 1980, 11, 562-568.

Ferguson D. J. P., Hutchison W. M. : An ultrastructural study of the early development and tissue cyst formation in the brains of mice. Parasitol. Res., 1987, 73, 483-491.

Frenkel J. K. : Toxoplasmosis: Parasite life cycle, pathology and immunology. In: The Coccidia, Eimeria, Isospora, Toxoplasma and related genera. D. M. HAmmond, P. L. Long, eds. University Park Press, Baltimore, 1973, 343-410.

Hoff R. L., Dubey J. P., Behbehani A. M., Frenkel J. K. : Toxoplasma gondii cysts in cell culture: new biologic evidence. J. Parasitol., 1977, 63, 1121-1124.

Jones T. C., BIEnz K. A., ERB P.: In vitro cultivation of Toxoplasma gondii cysts in astrocytes in the presence of gamma interferon. Inf. Immunol., 1986, 51, 147-156.

Martrou P., Pestre-Alexandre M., Loubet R., Nicolas J. A., Malinvaud G. : La toxoplasmose congénitale (note concernant un cas mortel). Limousin Med., 1965, 53, 3-7.

Matsubayashi H., Akao S. : Morphological studies on the development of the Toxoplasma cyst. Am. J. Trop. Med. Hyg., 1963, 1\%, 321-333.

Mehlhorn H., Frenkel J. K. : Ultrastructural comparison of cysts and zoïtes of Toxoplasma gondii, Sarcocystis muris, and Hammondia hammondi in skeletal muscle of mice. J. Parasitol., $1980,66,59-67$.

Nichols B. A., O'Connor G. R. : Penetration of mouse peritoneal macrophages by the protozo an Toxoplasma gondii. New evidence for active invasion and phagocytosis. Lab. Investig., $1981,44,324-335$.

Rondanelli E. G., Carosi G., Filice G., Benzi-Cipelli R., Carnevale G., Gorini G. : A comparative ultrastructural study of the low virulent strain PV-C1/81 and RH strain cultured in A9, VERO, SIRC cell lines. Bio. Res. Pregnancy, 1981, 3, 134-141.

Schwartzman J. D. : Quantitative comparison of infection of neural cell and fibroblast monolayers by two strains of Toxoplasma gondii (42 587). Proc. Soc. Exp. Biol. Med., 1987, 186, 75-78. 